\title{
Improved interpolating fields for hadrons at non-zero momentum
}

\author{
M. Della Morte ${ }^{1,2, a}$, B. Jäger ${ }^{1,2}$, T.D. Rae ${ }^{1}$, and H. Wittig ${ }^{1,2}$ \\ 1 PRISMA Cluster of Excellence, Institut für Kernphysik, Becher Weg 45, University of Mainz, D-55099 Mainz, Germany \\ 2 Helmholtz Institute Mainz, University of Mainz, D-55099 Mainz, Germany
}

Received: 3 August 2012 / Revised: 1 October 2012

Published online: 19 October 2012

(C) The Author(s) 2012. This article is published with open access at Springerlink.com Communicated by S. Hands

Abstract. We generalise Gaussian/Wuppertal smearing in order to produce non-spherical wave functions.
We show that we can achieve a reduction in the noise-to-signal ratio for correlation functions of certain
hadrons at non-zero momentum, while at the same time preserving a good projection on the ground state.

\section{Introduction}

In lattice QCD, masses, energies and transition matrix elements of asymptotic states with given quantum numbers are typically extracted from the Euclidean time dependence of suitable correlation functions. The contribution of the lowest state can be separated from those of other states by inserting the source fields at large-enough time distances. The associated statistical error can be estimated from the spectral properties of the theory $[1,2]$ and usually grows exponentially with the time separation ${ }^{1}$. In practice, this makes it very difficult to find a window where statistical and systematic errors are both under control. The use of smeared sources (i.e. interpolating fields with small overlaps on the excited states) ameliorates the situation, as the contribution from the lowest state is then expected to saturate the correlation function already at small Euclidean separations.

In general, the problem becomes more severe as nonzero spatial momenta are considered, as, for instance, in the computation of form factors, and it is therefore worth trying to optimise the smearing procedure depending on the kinematics. The most prominent example is given by the pion. At zero momentum the noise-to-signal ratio $\left(R_{\mathrm{NS}}\right)$ is expected to be constant as a function of the time $x_{0}$, whilst for $\mathbf{p} \neq 0$, from a generalization of the arguments presented in $[1,2]$, one expects

$$
R_{\mathrm{NS}}\left(x_{0}\right) \propto e^{\left(\sqrt{m_{\pi}^{2}+\mathbf{p}^{2}}-m_{\pi}\right) x_{0}}, \quad \text { for } \quad x_{0} \rightarrow \infty .
$$

\footnotetext{
a e-mail: morte@kph.uni-mainz.de

1 One exception is the pion channel (at zero momentum), for which the signal-to-noise ratio is asymptotically constant as a function of the source-sink separation.
}

Throughout the paper we will characterise the momentum of the pion (nucleon) by a vector of integers, $\mathbf{q}=$ $\left(q_{1}, q_{2}, q_{3}\right)$, where $\mathbf{p}=\mathbf{q} \frac{2 \pi}{L}$. In fig. 1 , we show the effective energies extracted from two-point correlation functions of fields interpolating pions at non-zero spatial momentum $\mathbf{q}$ for different values of $\mathbf{q}^{2}$. The plots refer to actual simulation results from measurements performed on an ensemble of $L / a=32, T / a=64, N_{\mathrm{f}}=2$ unquenched gauge configurations generated within the CLS initiative using 2 flavours of non-perturbatively $\mathrm{O}(a)$ improved Wilson fermions. Further numerical details can be found in $[3,4]$. In total, 168 configurations were used and for each of the configurations four evenly spaced source positions were utilised to improve the available statistics. We implemented Wuppertal smearing $[5,6]$ at the source and sink with parameters $\kappa=2.9$ and $n=140$, whose meaning will be explained in the next section. These values were chosen to maximise the length of the effective mass plateau (i.e. $\mathbf{p}^{2}=0$ ) for pions and nucleons and produce a Gaussian wave function with a radius of approximately $0.5 \mathrm{fm} \mathrm{[4].}$

The dramatic deterioration of the signal as $\mathbf{q}^{2}$ increases is quite striking. In fig. 2 , the function $\log R_{\mathrm{NS}}\left(x_{0}\right)$ from the same dataset is plotted as a function of $x_{0}$ for different values of $\mathbf{q}^{2}$ and it is compared to the expected asymptotic behaviour given in eq. (1). The asymptotic trend sets in quite early in the example and it is consistent with the noise being dominated by a zero-momentum two-pion state.

In the following, we will show how a significant improvement can be obtained by adopting non-spherical quark-smearing in order to interpolate boosted hadrons, instead of using the spherically symmetric function optimised for the zero-momentum case, as it is usually done. 
We underline that whilst this method provides a reduction of the noise in the measured quantities (the effective energies in this study), it does not achieve an exponential improvement of the noise-to-signal ratio.

A preliminary account of our work has been given in [7]. Here we study both the pion and nucleon channels. From the comparison it is clear that a much bigger gain in noise reduction for boosted pions can be achieved due to the fact that, for non-zero lattice momenta, the Lorentz factor $\gamma$ is much larger for pions than it is for nucleons.
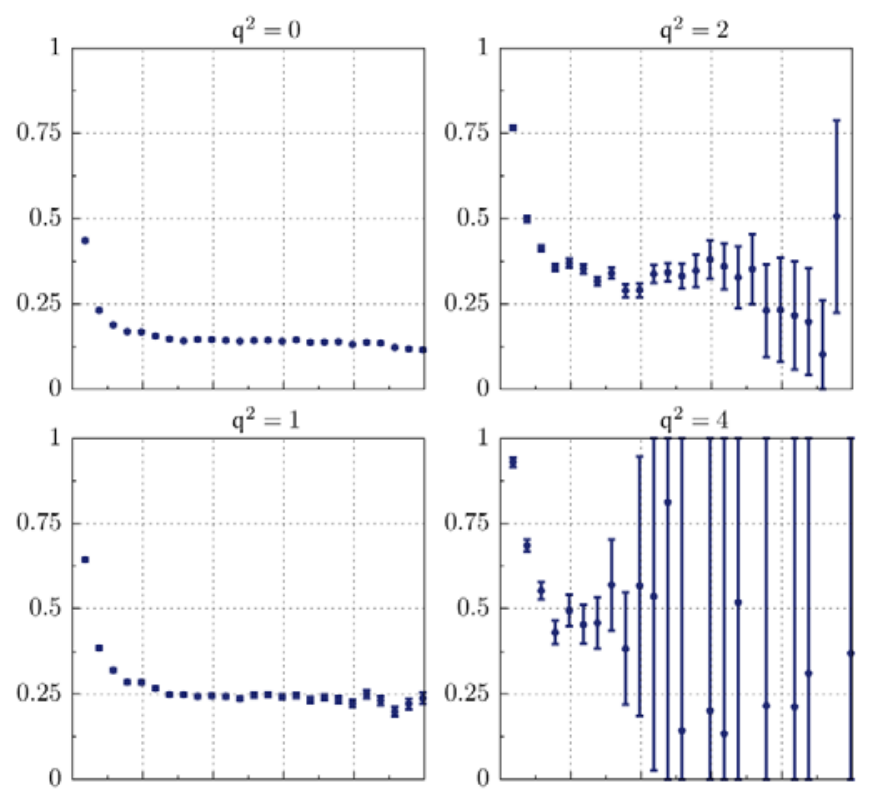

Fig. 1. Effective energies for pions at different values of $\mathbf{q}^{2}$.

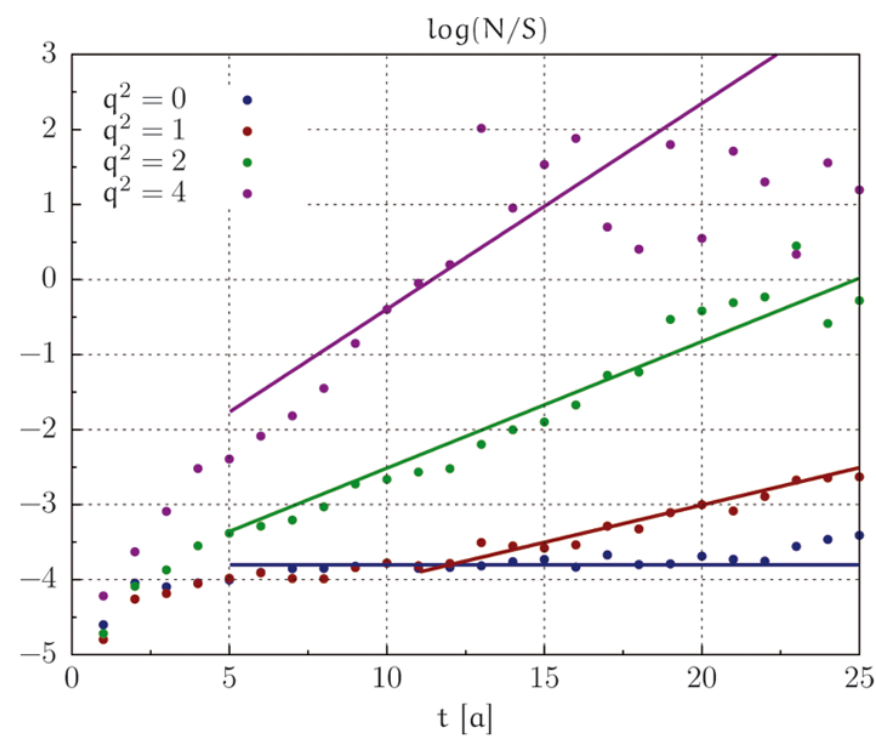

Fig. 2. Noise-to-signal ratio for different $\mathbf{q}^{2}$ plotted against the asymptotic behaviour of eq. (1).

\section{Anisotropic smearing for mesonic and baryonic 2-point functions}

We start by recalling the construction of Gaussian/Wuppertal smearing [5,6]. Given the hopping operator $H_{k}$ in direction $k$

$$
H_{k}\left(x, x^{\prime}\right)=U_{k}(x) \delta_{x^{\prime}, x+\hat{k}}+U_{k}^{\dagger}(x-\hat{k}) \delta_{x^{\prime}, x-\hat{k}},
$$

the smeared fermion field $\psi^{(\kappa)}$ is defined as

$$
\psi^{(\kappa)}(x)=(1+\kappa H)^{n} \psi(x),
$$

with

$$
H=H_{x}+H_{y}+H_{z} .
$$

In our generalization, we simply promote $\kappa$ and $H$ to spatial-vectors $\boldsymbol{\kappa}$ and $\mathbf{H}$ with components $x, y$ and $z$ and define

$$
\psi^{(\boldsymbol{\kappa})}(x)=(1+\boldsymbol{\kappa} \cdot \mathbf{H})^{n} \psi(x) .
$$

In this study, we fix $n=140$ and smear the gauge links in the hopping operator by applying one level of hypercubic smearing [8]. For the particular choice of smearing parameters, we follow the definition of "HYP2" links in [9]. We will consider two-point correlation functions of the type

$$
C(x)=\left\langle O\left(\psi^{(\boldsymbol{\kappa})}(x), \bar{\psi}^{(\boldsymbol{\kappa})}(x)\right) O^{\dagger}\left(\psi^{(\boldsymbol{\kappa})}(0), \bar{\psi}^{(\boldsymbol{\kappa})}(0)\right)\right\rangle,
$$

where the operator $O$ interpolates mesons or baryons and is therefore bi- or tri-linear in the fields $\psi^{(\boldsymbol{\kappa})}$ and $\bar{\psi}^{(\boldsymbol{\kappa})}$. Our goal is to reduce the zero-momentum component, which dominates the noise, or equivalently to enlarge the highmomentum components in the four-point correlation function describing the variance. This is achieved by spatially squeezing the quark wave function in the momentum direction. The choice is intuitively motivated by the physical picture that boosted hadrons are squeezed in the momentum direction because of the relativistic space contraction. As long as the size of the resulting wave function in the different directions is comparable to that of a boosted hadron we do not expect to spoil the good overlap with the ground state and therefore the fast approach to a plateau in the effective energy.

This generalization of Gaussian/Wuppertal smearing allows us to mix and parametrically deform point-like wave functions and broad Gaussian ones by tuning the components of $\boldsymbol{\kappa}$ in eq. (5). The first is known to give a better signal, especially at non-zero momentum, whereas the latter results in a better plateau [10]. We show in fig. 3 , the $y z$-section of a spherical wave function corresponding to $\kappa_{x}=\kappa_{y}=\kappa_{z}=2.9$ (left panel) as well as the $y z$-section of an anisotropic one, obtained using $\kappa_{x}=\kappa_{y}=2.9, \kappa_{z}=2.9 / 16$ (right panel). The lattice used is $32^{3} \times 64$ points with a lattice spacing of $0.063(2) \mathrm{fm}$ and a pion mass of roughly $450 \mathrm{MeV}[3,11]$. 

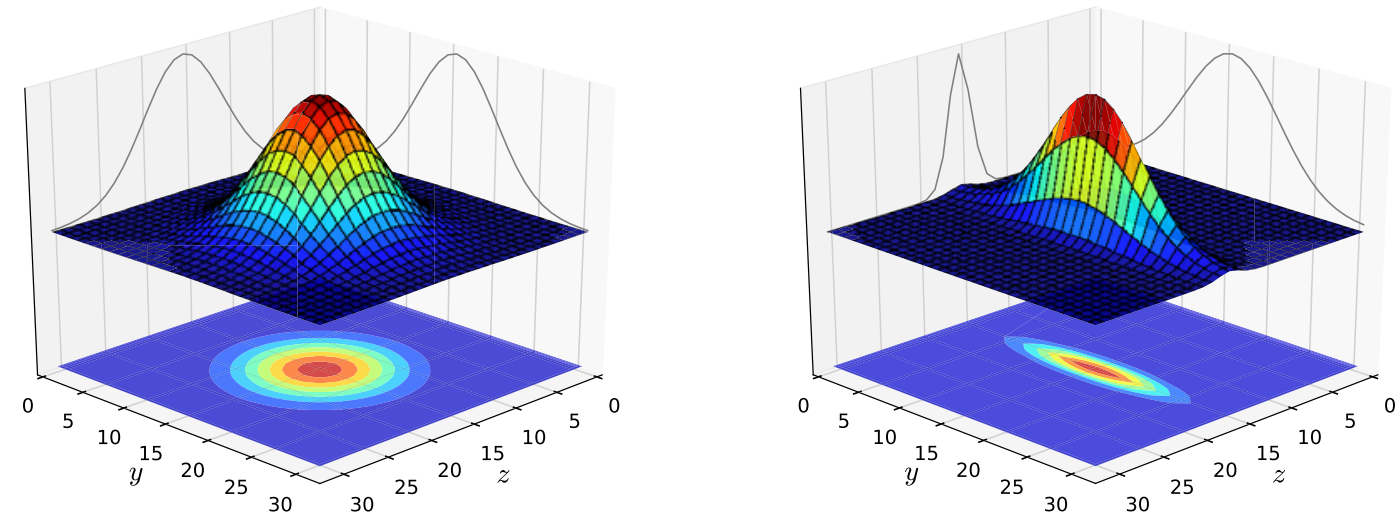

Fig. 3. The $y z$-section of a spherical wave function $\kappa_{x}=\kappa_{y}=\kappa_{z}=2.9$ (left panel) and an anisotropic wave function $\kappa_{x}=\kappa_{y}=2.9, \kappa_{z}=2.9 / 16$ (right panel).
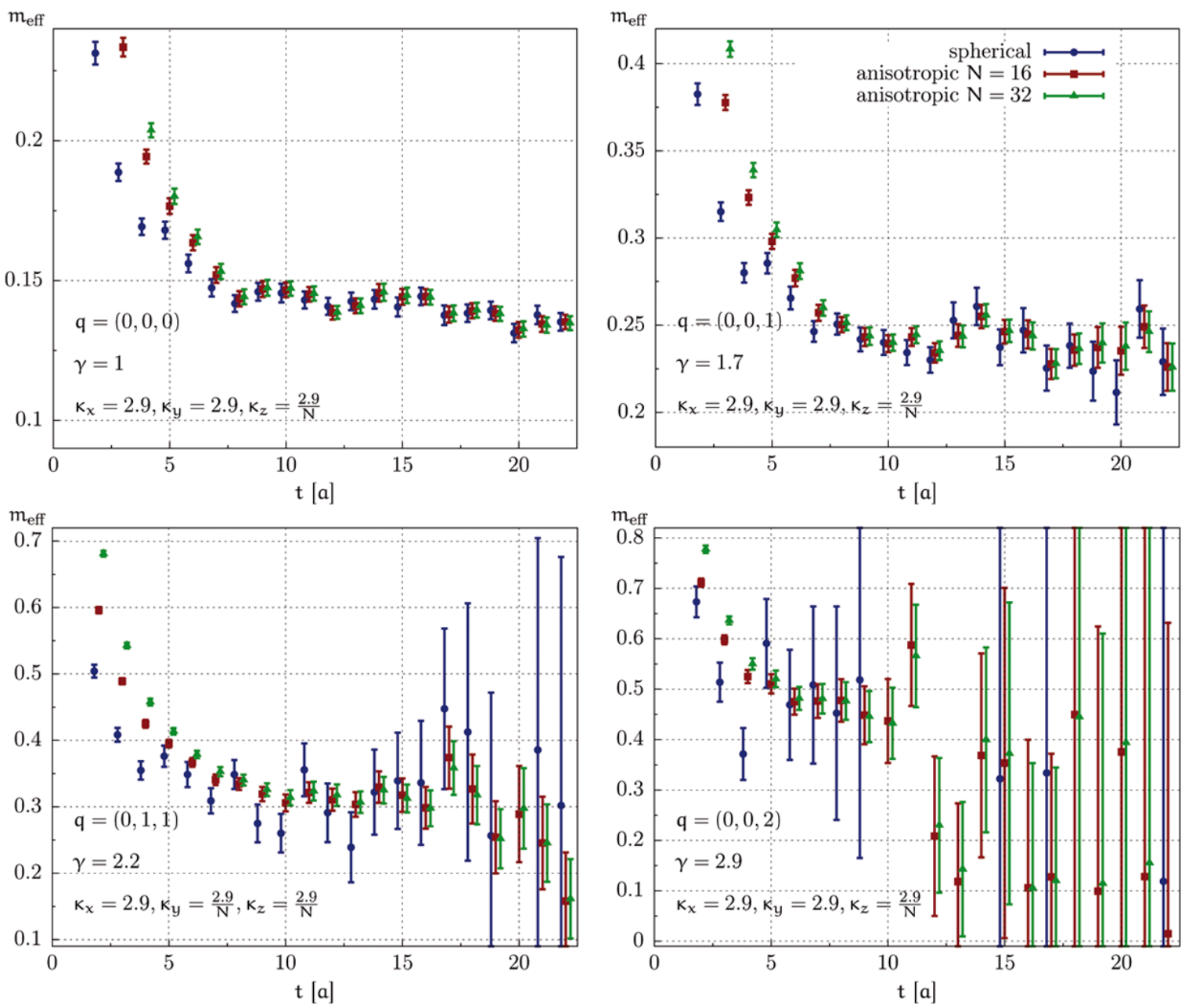

Fig. 4. Effective energies for the pion for different values of $\mathbf{q}^{2}$ using spherical (blue) and anisotropic (red and green) smearing. 
The anisotropic smearing is used so that the spatial squeezing is applied only to the directions with momenta. A numerical determination in one dimension demonstrated that for fixed $n$ in eq. (5), the size of the wave function in direction $l$ depends on $\kappa_{l}$ as roughly $\kappa^{1 / 5}$. Figure 4 compares the effect of spherical and anisotropic smearing on the effective energies for the first four Fourier momenta of the pion. This demonstrates a clear gain in the statistical signal, whilst preserving a good overlap with the ground state. The gain is more pronounced for an increasingly boosted pion. For instance, for $\mathbf{q}=(0,1,1)$ and $(0,0,2)$ the identification of the plateau and the statistical error is significantly improved. The plots display the direction of squeezing as well as the corresponding values of $\gamma$ to indicate the amount of boosting, where

$$
\gamma=\frac{\sqrt{m_{\mathrm{H}}^{2}+\mathbf{p}^{2}}}{m_{\mathrm{H}}},
$$

for a hadron $\mathrm{H}$.

The amount by which we spatially squeeze the directions with momenta should be dependent on the amount of boosting, $\gamma$. We checked this behaviour through implementing several choices for the wave function scaling $1 / N$, with $N=2,4,8,16$ and 32 . Figure 4 demonstrates that the improvement in statistical noise has saturated by $N=16$ and that any further improvement for $N=32$ is accompanied by a slightly reduced suppression of excited states. In order to further corroborate our intuitive argument we also checked that squeezing the $z$-direction

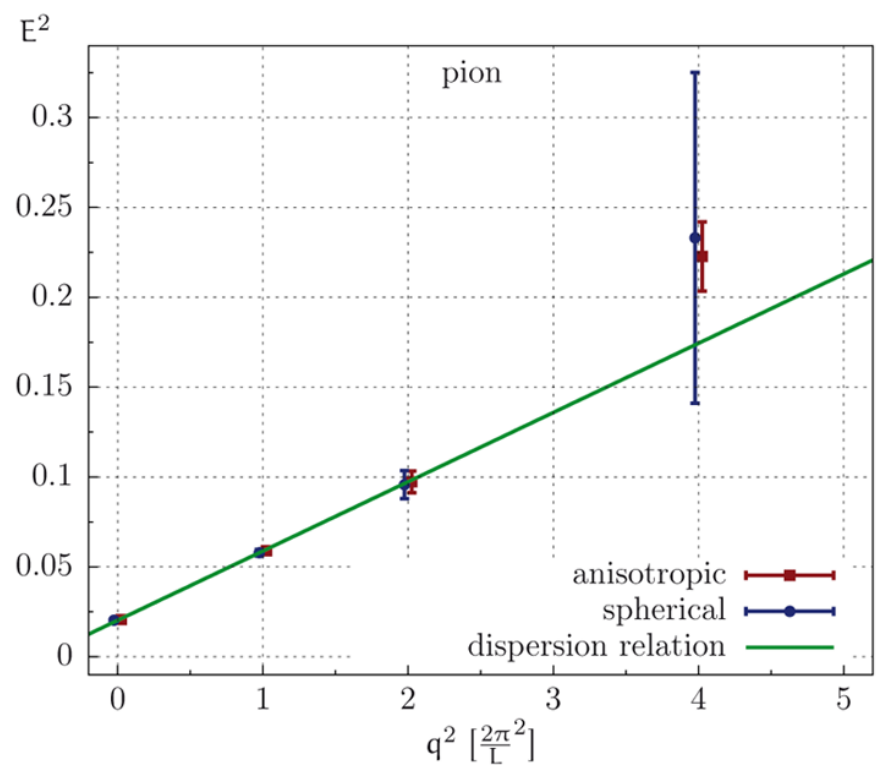

Fig. 5. The dispersion relation plotted against the extracted effective energies for spherical (blue) and anisotropic (red) smearing for the pion. The solid line denotes the continuum dispersion relation. for $\mathbf{q}=(1,0,0)$ or $(1,1,0)$, i.e. squeezing in an orthogonal direction to the momentum, does not provide a gain.

The gain achieved by anisotropic smearing is further illustrated in fig. 5, which shows the energies extracted from plateau fits to fig. 4. The fit-ranges are chosen to be the same for spherical and anisotropic smearing, and are determined through a $\chi^{2} /$ dof minimisation with a maximal plateau length. The continuum dispersion relation, determined from the physical pion mass, is overlaid to indicate the expected behaviour.

We apply the same treatment used for the pion to the nucleon, figures 6 and 7 . However, the signal-to-noise ratio is worse to begin with and so we are restricted from looking at significantly boosted nucleons. This means $\gamma \sim 1$ and therefore the gain is not as striking as for the pion. The most likely explanation for the observed difference between the dispersion relation and the data points is the presence of cutoff effects. The latter can be estimated from a comparison between the continuum dispersion relation and a linear fit of the three data points with the smallest momenta for both the spherical and anisotropic smearing. The difference of $4 \%$ amounts to less than two sigma and is therefore not statistically significant.

\section{Conclusions and outlook}

We have provided numerical evidence that by a simple generalization of Gaussian/Wuppertal smearing, which allows us to produce non-spherical quark wave fuctions, the noise-to-signal ratio in correlation functions describing the propagation of hadrons at non-zero momentum can be significantly reduced. The prescription yields a reduction of the statistical uncertainty without affecting the onset of a plateau in the effective energy and consequently the overlap of the wave function on the ground state. The strongest evidence is for the case of pions, as those are already highly boosted at small values of the typical lattice momenta. The comparison between pions and nucleons also indicates that the statistical gain mainly depends on the boosting factor, $\gamma$, rather than on the mass of the hadron. For this reason we believe this method to be even more effective as the chiral limit is approached. Of course, this expectation requires further studies, which we will consider in the future.

Recently, a very similar approach has been proposed in [12] for nucleon interpolating fields. In that case, only a small improvement has been observed, which is consistent with our findings.

The largest gain can probably be achieved by combining the approach with variational techniques, as in the Generalized Eigenvalue Problem method $[13,14]$. As a different smearing should in principle be adopted for each momentum, the use of all-to-all $[15,16]$ stochastic propagators could be advantageous in order to contain the numerical cost by reducing the number of inversions of the Dirac operator to be performed. We will explore these possibilities in future studies. 

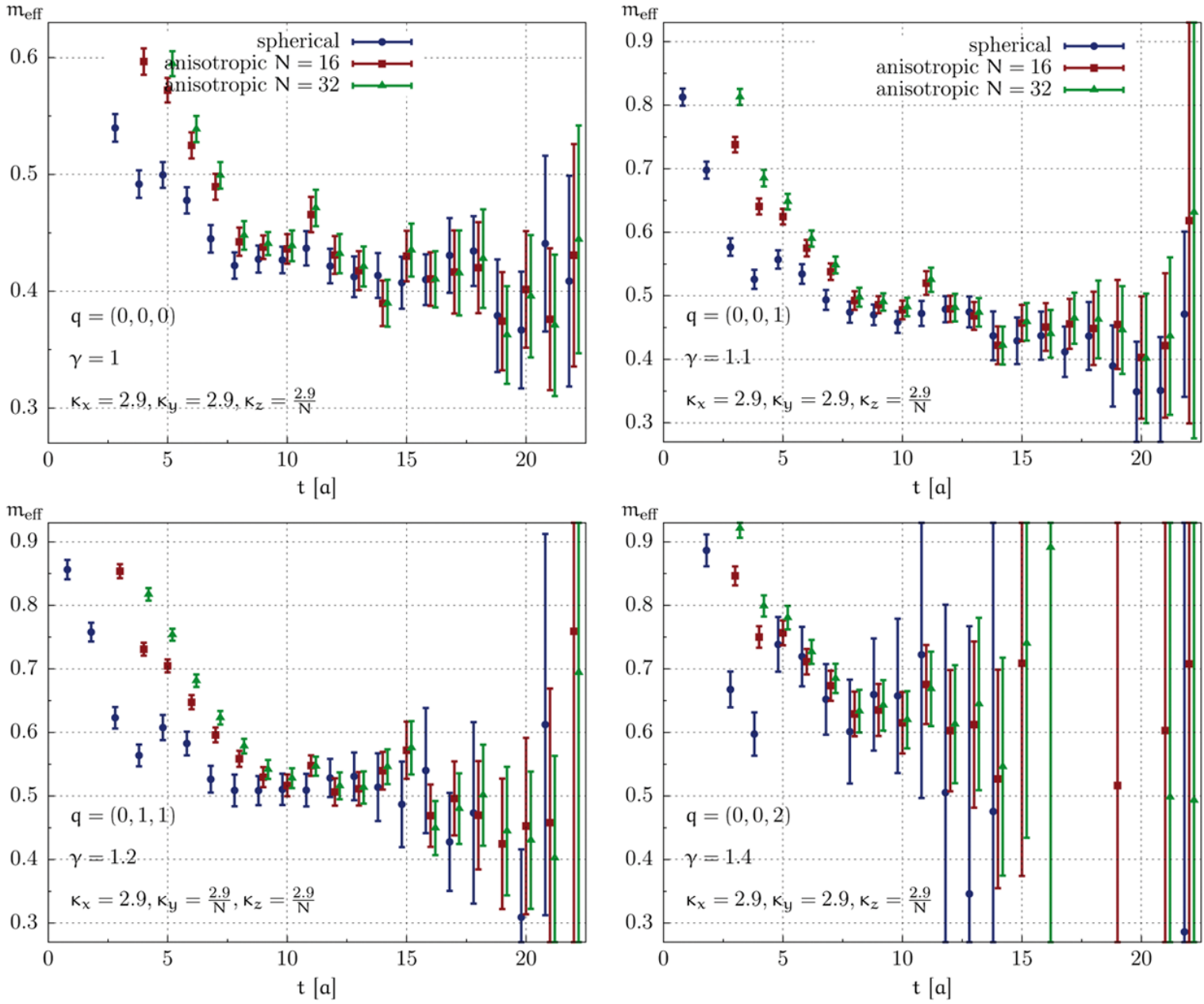

Fig. 6. Effective energies for the nucleon for different values of $\mathbf{q}^{2}$ using spherical (blue) and anisotropic (red and green) smearing.

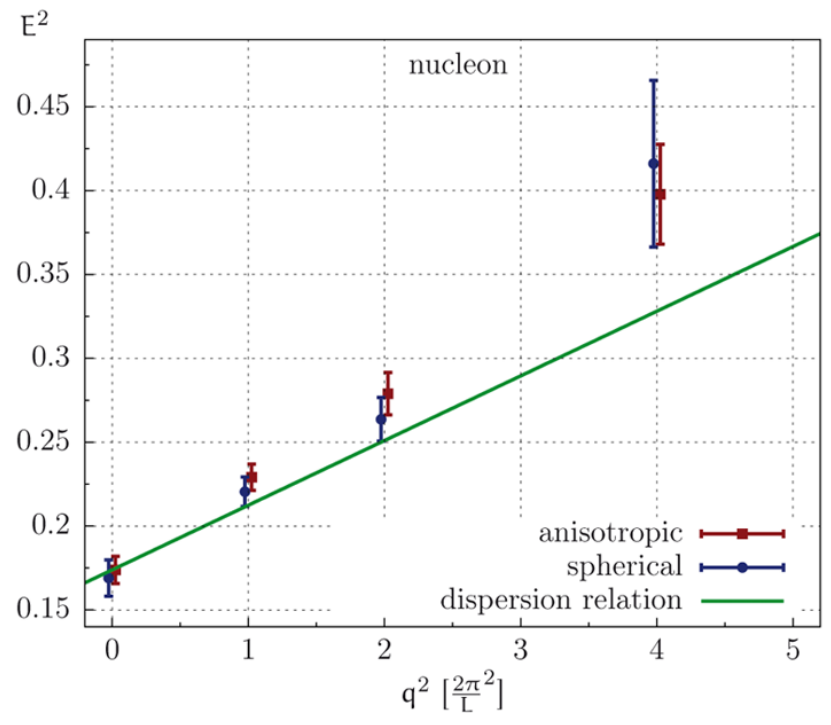

Fig. 7. The dispersion relation plotted against the extracted effective energies for spherical (blue) and anisotropic (red) smearing for the nucleon.
The main applications concern the determination of mesonic and baryonic form factors, e.g., for the $B \rightarrow$ $\pi l \nu$ process or for the nucleon form factors $G_{\mathrm{E}}$ and $G_{\mathrm{M}}$, on which we have recently presented preliminary results [17].

We are grateful for useful discussions with Bastian Brandt. Simulations were performed on the dedicated QCD platform "Wilson" at the Institute for Nuclear Physics, University of Mainz and on "JUGENE" at Forschungszentrum Jülich. We are indebted to the Institutes for their technical support and to the members of CLS for sharing the configurations. This work was partially supported by the Research Center EMG funded by Forschungsinitiative Rheinland-Pfalz. TR acknowledges support from DFG Grant HA4470/3-1

Open Access This is an open access article distributed under the terms of the Creative Commons Attribution License (http://creativecommons.org/licenses/by/3.0), which permits unrestricted use, distribution, and reproduction in any medium, provided the original work is properly cited. 


\section{References}

1. G. Parisi, Phys. Rep. 103, 203 (1984).

2. G.P. Lepage, TASI 89 Summer School, Boulder, CO, June 4-30, 1989.

3. P. Fritzsch, F. Knechtli, B. Leder, M. Marinkovic, S. Schaefer, R. Sommer, F. Virotta, Nucl. Phys. B 865, 397 (2012) arXiv: 1205.5380 [hep-lat].

4. S. Capitani, M. Della Morte, G. von Hippel, B. Jäger, A. Jüttner, B. Knippschild, H.B. Meyer, H. Wittig, arXiv:1205.0180 [hep-lat].

5. S. Güsken, U. Low, K.H. Mütter, R. Sommer, A. Patel, K. Schilling, Phys. Lett. B 227, 266 (1989).

6. S. Güsken, Nucl. Phys. Proc. Suppl. 17, 361 (1990).

7. M. Della Morte, B. Jäger, T. Rae, H. Wittig, PoS LATTICE 2012, 260 (2012) poster presented by BJ and TR at "Lattice 2012", Cairns, Australia, June 26, 2012.

8. A. Hasenfratz, F. Knechtli, Phys. Rev. D 64, 034504 (2001) hep-lat/0103029.

9. M. Della Morte, A. Shindler, R. Sommer, JHEP 08, 051 (2005) hep-lat/0506008.
10. H.-W. Lin, S.D. Cohen, R.G. Edwards, K. Orginos, D.G. Richards, arXiv:1005.0799 [hep-lat].

11. S. Capitani, M. Della Morte, G. von Hippel, B. Knippschild, H. Wittig, PoS LATTICE 2011, 145 (2011) arXiv:1110.6365 [hep-lat].

12. D.S. Roberts, W. Kamleh, D.B. Leinweber, M.S. Mahbub, B.J. Menadue, arXiv:1206.5891 [hep-lat].

13. M. Lüscher, U. Wolff, Nucl. Phys. B 339, 222 (1990).

14. B. Blossier, M. Della Morte, G. von Hippel, T. Mendes, R. Sommer, JHEP 04, 094 (2009) arXiv:0902.1265 [hep-lat].

15. J. Foley, K. Jimmy Juge, A. O'Cais, M. Peardon, S.M. Ryan, J.-I. Skullerud, Comput. Phys. Commun. 172, 145 (2005) hep-lat/0505023.

16. E. Endress, A. Jüttner, H. Wittig, arXiv:1111.5988 [heplat].

17. S. Capitani, M. Della Morte, G. von Hippel, B. Jäger, B. Knippschild, H.B. Meyer, H. Wittig, PoS LATTICE 2012, 177 (2012) talk presented by TR at "Lattice 2012", Cairns, Australia, June 28, 2012. 